\title{
Effects of intrauterine infection in different periods on the placenta and endometrial blood vessel formation of pregnant mice and the growth and development of fetal rats
}

\author{
Xiao-li Geng', Ya-xuan Zhang*2, Qi-zhi Ren ${ }^{3}$ \\ ${ }^{1}$ Department of Obstetrics and Gynecology, the Third Affiliated Hospital of Inner Mongolia Medical University, Baotou, Inner \\ Mongolia, China \\ ${ }^{2}$ Department of Neurosurgery, the First Affiliated Hospital of Baotou Medical College, Baotou, Inner Mongolia, China \\ ${ }^{3}$ Department of Obstetrics and Gynecology, Baogang Hospital, Baotou, Inner Mongolia, China
}

Received: April 15, 2021

DOI: $10.5430 /$ dcc.v8n2p18
Accepted: July 30, 2021

Online Published: August 22, 2021

\begin{abstract}
Objective: To investigate the effects of intrauterine infection in different periods on the placenta and endometrial blood vessel formation of pregnant rats and the growth and development of fetal rats.

Methods: According to the random number table method, 32 pregnant rats were divided into the early infection group, the mid-term infection group, the late infection group and the control group, with 8 rats in each group. On the $3^{\text {rd }}, 9^{\text {th }}$ and $15^{\text {th }}$ day of pregnancy, lipopolysaccharide was injected intraperitoneally to construct intrauterine infection models. The pregnant rats in the control group were intraperitoneally injected with the same dose of $0.9 \%$ sodium chloride solution. On the $18^{\text {th }}$ day of pregnancy, the inflammatory factors [interleukin-6 (IL-6), tumor necrosis factor- $\alpha$ (TNF- $\alpha$ )], the blood vessel density of placenta and endometrium in the placental tissues of pregnant rats, dead fetus + absorbed fetus, the inflammatory factors IL-6, TNF- $\alpha$ and oxidation reaction indicators [malondialdehyde (MDA) and myeloperoxidase (MPO)] in the fetal rat lung and brain tissues were detected.

Results: The changing trend of IL- 6 and TNF- $\alpha$ levels in the placental tissues of pregnant rats with intrauterine infection in different periods was: the control group $<$ the late infection group $<$ the mid-term infection group $<$ the early infection group, the differences were statistically significant $(p<.05)$. The changing trend of fetal rat weight, placental weight and placental coefficient in the intrauterine infection groups in different periods was: the control group $>$ the late infection group $>$ the mid-term infection group $>$ the early infection group, the differences were statistically significant $(p<.05)$. The blood vessel density of placenta and endometrium, the mean number of fetuses, brain coefficient and lung coefficient in the late infection group were significantly increased in comparison with the early infection group and the mid-term infection group. The total number and the ratio of dead fetus + absorbed fetus, the levels of IL-6, TNF- $\alpha$, MDA and MPO in brain and lung tissues were significantly reduced, and the differences were statistically significant $(p<.05)$. The blood vessel density of placenta and endometrium, brain coefficient and lung coefficient of pregnant rats in the mid-term infection group were significantly increased in comparison with the early infection group, and the differences were statistically significant $(p<.05)$. There was no statistically significant difference in the other indicators between the two groups $(p>.05)$.

Conclusions: Intrauterine infection in different periods can inhibit placental and endometrial angiogenesis, and affect the survival
\end{abstract}

*Correspondence: Ya-xuan Zhang; Email: jinli81636772@163.com; Address: Department of Neurosurgery, the First Affiliated Hospital of Baotou Medical College, Baotou, Inner Mongolia 014010, China. 
rate of fetal rats and the growth and development of brain and lung. The reason may be related to the aggravation of fetal inflammatory responses and oxidative stress. The earlier the intrauterine infection occurs, the severer the adverse effects on the fetal rats will be.

Key Words: Pregnant rats, Intrauterine infection, Inflammation, Endometrium, Blood vessel formation

\section{INTRODUCTION}

Intrauterine infection (IAI) refers to the infection in amniotic fluid, fetal membrane (amniotic membrane, chorion), placenta and fetus caused by the invasion of pathogenic microorganisms into the amniotic cavity, which is also called intraamniotic infectious syndrome (IAIS). ${ }^{[1]}$ It is reported in the literature that the incidence of intrauterine infection ranges from $4.0 \%$ to $10.0 \%$, and is gradually increasing overall. ${ }^{[2]}$ IAI involves a variety of pathogens, with a complicated pathogenesis. The invasion of pathogenic microorganisms from the vagina, the neck of uterus and the blood into the uterus can lead to a series of adverse outcomes. The Intrauterine infection in the first trimester of pregnancy can result in abortion; the infection in the second trimester of pregnancy can arouse fetal growth restriction, premature rupture of fetal membranes, premature delivery, fetal anomaly, neonatal infection and so on; ${ }^{[3,4]}$ in the last trimester of pregnancy, it can cause premature delivery, abortion, premature rupture of fetal membranes, neonatal infection, neonatal brain damage and other severe syndromes. IAI is one of the most important factors for perinatal and maternal death. ${ }^{[5]}$ Therefore, it is of great importance to make an early diagnosis of intrauterine infection during pregnancy and give a timely symptomatic treatment in order to reduce perinatal anomaly, premature delivery, death and other syndromes. Placenta is a pregnancyspecific maternal-fetal organ for the exchange of substances. The placenta and the endometrium with normal vascular function can maintain the normal pregnancy, the fetal growth and development and the onset of labor. ${ }^{[6]}$ As cytokines can go through the placenta and the blood-brain barrier to affect the fetal development, the adverse effects of IAI on the fetal development are mediated by proinflammatory cytokines to a large extent. ${ }^{[7]}$ In recent years, more literatures have confirmed that IAI can lead to the inflammatory responses, fetal anomaly, dead fetus, fetal dysplasia and so on. ${ }^{[8,9]}$ However, there are few researches on the effects of IAI in different periods of pregnancy on pregnant and fetal rats. Pyrexia and the up-regulated expression level of inflammatory factors are commonly-seen immune responses, which play an important role in assisting in the anti-infective process. In this research, the intraperitoneal injection of lipopolysaccharide in the process of pregnancy is conducted to establish the rat IAI models in different trimesters of pregnancy. By measur- ing the related indicators about the serum, the placenta and the endometrium in pregnant and fetal rats, this research is designed to explore the effects of IAI in different periods of pregnancy. It is reported as follows.

\section{MATERIALS AND METHODS}

\subsection{Experimental animals}

Clean SD rats, provided by Laboratory Animal Center of the Third Affiliated Hospital of Inner Mongolia Medical University [Certification No.: SYXK (M2020-0003)]. There were 36 female rats (weighted 240-280 g) and 12 male rats (weighed 300-350 g). The feeding conditions of rats were: the temperature was kept at $(25 \pm 1)^{\circ} \mathrm{C}$, the relative humidity was controlled at $50 \%-55 \%$, and all rats had free access to water and feed. Daily periodicity was set as $12 \mathrm{~h} / 12 \mathrm{~h}$, with continuous lighting and excellent ventilation. The research program, the experimental contents and the operation procedures were all based on the ethical requirements for animals.

\subsection{Main reagents and instruments}

Lipopolysaccharide was provided by SIGMA (USA); interleukin-6 (IL-6), tumor necrosis factor- $\alpha$ (TNF- $\alpha$ ), malondialdehyde (MDA), myeloperoxidase (MPO) and enzyme-linked immunosorbent assay (ELISA) kits were purchased from Shanghai Jianglai Biotechnology Co., Ltd.; Hematoxylin-Eosin (H\&E) kits were merchandised from MesGen Biotechnology (China); the microplate reader was bought from Molecular Devices, LLC. (Shanghai, China); the fluorescent microscope and the image-forming system were procured from Nikon Corporation (Japan).

\subsection{Grouping and administration method}

At 20:00, the female rats cohabited with the male rats at the ratio of $3: 1$. The next morning, vaginal smears were examined at 8:00 in order to identify the presence of sperms. If the result was positive, it was considered as the first day of pregnancy. The pregnant rats were fed separately. According to the random number table method, 32 pregnant rats were divided into the early infection group $(n=8)$, the mid-term infection group $(n=8)$, the late infection group $(n=8)$ and the control group $(n=8)$. The IAI infection groups were given intraperitoneal injection of lipopolysaccharide $(350 \mu \mathrm{g} / \mathrm{kg})$ on the $3^{r d}, 9^{t h}$ and $15^{\text {th }}$ day of pregnancy respectively, in 
order to construct intrauterine infection models. Successful modeling of IAI pregnant rats can be confirmed by the rat weight monitoring and the pathological observation of the damaged brain tissues in the modeling group of rats. ${ }^{[10]}$ The pregnant rats in the control group were intraperitoneally injected with the same dose of $0.9 \%$ sodium chloride solution.

\subsection{Indicator observation}

\subsubsection{The measurement of inflammatory factors in the serum}

On the $18 \mathrm{~d}$ of pregnancy (or upon premature delivery), each group of rats were taken blood by means of docking. ELISA was used to measure the contents of IL- $1 \beta$, IL- 6 and TNF- $\alpha$ in the serum. The instructions of ELISA kit were strictly followed to conduct the operation procedures. The microplate reader was used to measure OD value (wave length $450 \mathrm{~nm}$ ) The corresponding concentration was calculated according to the standard curve.

\subsubsection{The measurement of the fetal rat weight and placen- tal coefficient}

After each group of rats were sacrificed by cervical dislocation on the $18 \mathrm{~d}$ of pregnancy (or upon premature delivery), fetuses and placentae were taken out, with the blood wiped out. After being weighed, the fetal rats were also sacrificed by cervical dislocation. The placentae were rinsed out by use of $0.9 \%$ sodium chloride solution and then placed on the filter paper for the use of weighing. The calculation method was as follows: placental coefficient = placental weight $/$ fetal weight.

\subsubsection{The measurement of inflammatory factors in the pla- cental tissues}

After placenta weighing, a small number of tissues were taken from the placenta randomly chosen from one of the three gestational sacs. ELISA method was used to measure the contents of IL- 6 and TNF- $\alpha$.

\subsubsection{The measurement of the blood vessel density of pla- centa and endometrium}

The placental and the endometrial tissues were separated from the pregnant rats, rinsed out by use of $0.9 \%$ sodium chloride solution. After the conventional dewaxing, the tissue section was stained with BS-lectin and then photomicrographed. According to the point-counting method, IPP 6.0 software was used to calculate the blood vessel density of placenta and endometrium in pregnant rats.

\subsubsection{The measurement of the fetal indicators}

(1) The ratio of dead fetus + absorbed fetus: After the rats got infected, the delivery and the survival rate of the fetal rats were observed and recorded accordingly. (2) The measurement of brain and lung coefficients: The brain and the lung tissues were rinsed out by use of $0.9 \%$ sodium chloride solution and then placed on the filter paper for the use of weighing. The changing trend of brain and lung coefficients were monitored. Brain coefficient $=$ brain weight $/$ fetal weight, lung efficient = lung weight/fetal weight. (3) The measurement of cytokines in brain tissues: After brain tissues were acquired, ELISA method was used to measure the contents of IL-6, TNF- $\alpha$, MDA and MPO. The instructions were strictly followed to conduct the operation procedures. The microplate reader was used to measure OD value (wave length $450 \mathrm{~nm}$ ). The corresponding concentration was calculated according to the standard curve. (4) The measurement of cytokines in lung tissues: After a small amount of lung tissues were acquired, ELISA method was used to measure the contents of IL- 6 and TNF- $\alpha$; the rest of lung tissues were fixed in $4 \%$ paraformaldehyde solution for $24 \mathrm{~h}$, and then dehydrated, embedded to make the preparation of paraffin sections, with a thickness of $4 \mu \mathrm{m}$. HE staining method was used to monitor the changes of vesicle-like structures, alveolar septum and alveoli.

\subsection{Statistical treatment}

SPSS 16.0 statistical software was applied to the statistical analysis of the acquired data. The measurement data were represented by mean \pm standard deviation $(\bar{x} \pm s)$, Oneway ANOVA was applied to the comparison among multiple groups, LSD test and two-sample group $t$-test were used in the comparison between two groups. The difference $(p<.05)$ was of statistical significance.

\section{RESULTS}

3.1 The levels of IL-6 and TNF- $\alpha$ in the placental tissues from each group of pregnant rats

The levels of IL- 6 and TNF- $\alpha$ in the placental tissues from the pregnant rats in the early infection group, the mid-term infection group and the late infection group were all significantly higher than those in the control group. Meanwhile, the levels of IL-6 and TNF- $\alpha$ in the placental tissues of the pregnant rats in the late infection group were lower than those in the mid-term infection group and the early infection group. These indicators in the mid-term infection group were lower than those in the early infection group. The differences were statistically significant $(p<.05)$. See Table 1 for details.

\subsection{The comparison in fetal weight, placental weight and placental coefficient among these groups of preg- nant rats}

Fetal weight, placental weight and placental coefficient in the early infection group, the mid-term infection group and the late infection group were obviously higher than those in the control group. Meanwhile, these indicators in the late 
infection group were higher than those in the mid-term in- infection group. The differences were statistically significant fection group and the early infection group. In the mid-term $\quad(p<.05)$. See Table 2 for details. infection group, they were also higher than those in the early

Table 1. The levels of IL-6 and TNF- $\alpha$ in the placental tissues from each group of pregnant rats $(\bar{x} \pm \mathrm{s})$

\begin{tabular}{|c|c|c|c|}
\hline Group & The Number of Rats & IL-6 (pg/ml) & TNF- $\alpha(\mu \mathrm{g} / \mathrm{ml})$ \\
\hline Control Group & 8 & $7.09 \pm 0.83$ & $0.28 \pm 0.04$ \\
\hline Early Infection Group & 8 & $29.11 \pm 0.85^{*}$ & $0.83 \pm 0.03^{*}$ \\
\hline Mid-term Infection Group & 8 & $18.44 \pm 1.30^{* \&}$ & $0.75 \pm 0.06^{* \&}$ \\
\hline Late Infection Group & 8 & $10.69 \pm 0.85^{* \& \#}$ & $0.43 \pm 0.06^{*} \& \#$ \\
\hline$F$ Value & & 794.910 & 223.973 \\
\hline$p$ Value & & $<0.001$ & $<0.001$ \\
\hline
\end{tabular}

Table 2. The comparison in fetal weight, placental weight and placental coefficient among these groups of pregnant rats $(\bar{x} \pm \mathrm{s})$

\begin{tabular}{lllll}
\hline Group & The Number of Rats & Fetal Weight (mg) & Placental Weight (mg) & Placental Coefficient \\
\hline Control Group & 8 & $819.76 \pm 10.75$ & $116.89 \pm 14.82$ & $0.14 \pm 0.02$ \\
Early Infection Group & 8 & $586.96 \pm 3.85^{*}$ & $43.64 \pm 4.16^{*}$ & $0.07 \pm 0.01^{*}$ \\
Mid-term Infection Group & 8 & $655.36 \pm 15.99^{* \&}$ & $67.76 \pm 4.14^{* \&}$ & $0.10 \pm 0.02^{* \&}$ \\
Late Infection Group & 8 & $800.85 \pm 11.00^{* \& \#}$ & $100.27 \pm 5.70^{* \& \#}$ & $0.12 \pm 0.01^{* \& \#}$ \\
$F$ Value & & 805.554 & 120.052 & 28.533 \\
$p$ Value & & $<.001$ & $<.001$ & $<.001$ \\
\hline
\end{tabular}

Note. ${ }^{*}$ In comparison with the control group, $p<.05$; ${ }^{\&}$ in comparison with the early infection group, $p<.05$; ${ }^{*}$ in comparison with the late infection group, $p<.05$.

\subsection{The measurement of the blood vessel density of pla- centa and endometrium}

The blood vessel density of placenta and endometrium in the early infection group and the mid-term infection group was lower than that in the control group respectively, and the differences were of statistical significance $(p<.05)$. There was no statistically significant difference between the late infection group and the control group $(p>.05)$. In the late infection group, the blood vessel density of placenta and endometrium was obviously higher than that in the mid-term infection group and the early infection group respectively, and in the mid-term infection group, it was obviously higher than that in the early infection group. The differences were of statistical significance $(p<.05)$. See Table 3 for details.

\subsection{The comparison in the survival condition, brain and lung function of fetuses among the groups of preg- nant rats}

In the early infection group and the mid-term infection group, the mean number of fetuses, brain coefficient and lung coefficient were all remarkably lower than those in the control group, and the total number and the ratio of dead fetus + absorbed fetus were significantly higher than those in the control group. The differences were statistically significant $(p<.05)$. In the late infection group, the mean number of fetuses, brain coefficient and lung coefficient were obviously higher than those in the early infection group and the midterm infection group, and the total number and the ratio of dead fetus + absorbed fetus were significantly lower those in the early infection group and the mid-term infection group. The differences were of statistical significance $(p<.05)$. There was no statistically significant difference in the mean number of fetuses, the total number and the ratio of dead fetus + absorbed fetus between the early infection group and the mid-term infection group $(p>.05)$. However, brain coefficient and lung coefficient in the mid-term infection group were significantly higher than those in the early infection group, and the differences were of statistical significance ( $p$ $<.05)$. See Table 4 for details.

\subsection{The comparison in the levels of IL-6, TNF- $\alpha$, MDA and MPO in brain and lung tissues from each group of fetal rats}

In the early infection group, the mid-term infection group and the late infection group, the levels of IL-6, TNF- $\alpha$, MDA and MPO in brain and lung tissues from each group of fetal rats were obviously higher than those in the control group, and the differences were statistically significant $(p<.05)$; 
however, in the late infection group, these indicators were ob- significant difference in the levels of IL-6, TNF- $\alpha$, MDA and viously lower than those in the early infection group and the middle infection group, and the differences were of statistical significance $(p<.05)$. Nevertheless, there was no statistically MPO in brain and lung tissues from each group of fetal rats between the early infection group and the mid-term infection group $(p>.05)$. See Table 5 and Table 6 for details.

Table 3. The comparison in the blood vessel density of placenta and endometrium $\left(\mathrm{mm}^{-2}, \bar{x} \pm \mathrm{s}\right)$

\begin{tabular}{llll}
\hline Group & The Number of Rats & Placental Blood Vessel Density & Endometrial Blood Vessel Density \\
\hline Control Group & 8 & $95.1 \pm 12.2$ & $70.8 \pm 8.1$ \\
Early Infection Group & 8 & $54.8 \pm 10.4^{*}$ & $36.4 \pm 5.9^{*}$ \\
Mid-term Infection Group & 8 & $72.6 \pm 9.0^{* \&}$ & $52.0 \pm 5.4^{* \&}$ \\
Late Infection Group & 8 & $93.9 \pm 8.2^{\& \#}$ & $69.3 \pm 9.1^{\& \#}$ \\
$F$ Value & & 29.159 & 39.727 \\
$p$ Value & $<.001$ & $<.001$ \\
\hline Note. ${ }^{*}$ In comparison with the control group, $p<.05$; $^{*}$ in comparison with the early infection group, $p<.05 ;{ }^{*}$ in comparison with the mid-term infection group, $p<.05$.
\end{tabular}

Table 4. The comparison in the mean number of fetuses, the total number and the ratio of dead fetus + absorbed fetus among the groups of pregnant rats $(\bar{x} \pm \mathrm{s})$

\begin{tabular}{lllllll}
\hline \multirow{2}{*}{ Group } & $\begin{array}{l}\text { The } \\
\text { Number of } \\
\text { Rats }\end{array}$ & $\begin{array}{l}\text { The Mean } \\
\text { Number of } \\
\text { Fetuses (n) }\end{array}$ & $\begin{array}{l}\text { The Total Number } \\
\text { of Dead Fetus }+ \\
\text { Absorbed Fetus (n) }\end{array}$ & $\begin{array}{l}\text { The Ratio of Dead } \\
\text { Fetus + Absorbed } \\
\text { Fetus (\%) }\end{array}$ & $\begin{array}{l}\text { Brain } \\
\text { Coefficient }\end{array}$ & $\begin{array}{l}\text { Lung } \\
\text { Coefficient }\end{array}$ \\
\hline Control Group & 8 & $12.0 \pm 2.4$ & $0.3 \pm 0.1$ & $1.3 \pm 0.5$ & $0.23 \pm 0.02$ & $0.31 \pm 0.01$ \\
Early Infection Group & 8 & $7.1 \pm 2.1^{*}$ & $1.1 \pm 0.2^{*}$ & $16.0 \pm 2.2^{*}$ & $0.07 \pm 0.01^{*}$ & $0.13 \pm 0.01^{*}$ \\
Mid-term Infection Group & 8 & $8.9 \pm 2.0^{*}$ & $0.9 \pm 0.2^{*}$ & $9.4 \pm 2.4^{*}$ & $0.13 \pm 0.02^{* \&}$ & $0.20 \pm 0.02^{* \&}$ \\
Late Infection Group & 8 & $11.6 \pm 3.1^{\& \#}$ & $0.4 \pm 0.1^{\& \#}$ & $2.7 \pm 0.5^{\& \#}$ & $0.22 \pm 0.01^{\& \#}$ & $0.29 \pm 0.02^{\& \#}$ \\
$F$ Value & & 7.240 & 47.733 & 131.892 & 186.400 & 222.667 \\
$p$ Value & & .001 & $<.001$ & $<.001$ & $<.001$ & $<.001$ \\
\hline
\end{tabular}

Note. ${ }^{*}$ In comparison with the control group, $p<.05 ;{ }^{*}$ in comparison with the early infection group, $p<.05 ;{ }^{*}$ in comparison with the mid-term infection group, $p<.05$.

Table 5. The comparison in the levels of IL-6, TNF- $\alpha$, MDA and MPO in brain tissues from each group of fetal rats $(\bar{x} \pm \mathrm{s})$

\begin{tabular}{llllll}
\hline Group & The Number of Rabbits & IL-6 $(\mathbf{p g} / \mathbf{m l})$ & TNF- $\boldsymbol{\alpha}(\boldsymbol{\mu g} / \mathbf{m l})$ & MDA $(\mathbf{n m o l} / \mathbf{g})$ & MPO $(\mathbf{U} / \mathbf{g})$ \\
\hline Control Group & 8 & $4.34 \pm 0.23$ & $0.17 \pm 0.02$ & $84.54 \pm 1.76$ & $0.36 \pm 0.03$ \\
Early Infection Group & 8 & $15.52 \pm 1.35^{*}$ & $0.49 \pm 0.03^{*}$ & $166.16 \pm 10.29^{*}$ & $2.08 \pm 0.44^{*}$ \\
Mid-term Infection Group & 8 & $14.43 \pm 2.20^{*}$ & $0.44 \pm 0.05^{*}$ & $167.16 \pm 12.45^{*}$ & $2.06 \pm 0.29^{*}$ \\
Late Infection Group & 8 & $9.50 \pm 1.23^{* \& \#}$ & $0.29 \pm 0.02^{* \& \#}$ & $123.41 \pm 6.04^{* * \#}$ & $1.32 \pm 0.18^{* \& \#}$ \\
$F$ Value & & 102.138 & 161.714 & 166.332 & 67.701 \\
$p$ Value & & $<.001$ & $<.001$ & $<.001$ & $<.001$ \\
\hline
\end{tabular}

Note. ${ }^{*}$ In comparison with the control group, $p<.05 ;{ }^{*}$ in comparison with the early infection group, $p<.05 ;{ }^{*}$ in comparison with the mid-term infection group, $p<.05$.

Table 6. The comparison in the levels of IL-6, TNF- $\alpha$, MDA and MPO in lung tissues from each group of fetal rats $(\bar{x} \pm \mathrm{s})$

\begin{tabular}{llllll}
\hline Group & The Number of Rats & IL-6 $(\mathbf{p g} / \mathbf{m l})$ & TNF- $\boldsymbol{\alpha}(\boldsymbol{\mu g} / \mathbf{m l})$ & MDA $(\mathbf{n m o l} / \mathbf{g})$ & MPO $(\mathbf{U} / \mathbf{g})$ \\
\hline Control Group & 8 & $5.56 \pm 0.17$ & $0.24 \pm 0.03$ & $90.26 \pm 6.30$ & $0.54 \pm 0.08$ \\
Early Infection Group & 8 & $17.49 \pm 1.27^{*}$ & $0.63 \pm 0.12^{*}$ & $190.43 \pm 15.71^{*}$ & $2.55 \pm 0.32^{*}$ \\
Mid-term Infection Group & 8 & $16.60 \pm 1.31^{*}$ & $0.51 \pm 0.13^{*}$ & $184.55 \pm 11.09^{*}$ & $2.42 \pm 0.51^{*}$ \\
Late Infection Group & 8 & $6.05 \pm 0.45^{* * \#}$ & $0.28 \pm 0.04^{*} \& \#$ & $275.16 \pm 14.79^{*} \& \#$ & $6.85 \pm 0.60^{*} \& \#$ \\
$F$ Value & & 380.043 & 32.852 & 290.921 & 312.882 \\
$p$ Value & & $<.001$ & $<.001$ & $<.001$ & $<.001$ \\
\hline
\end{tabular}

Note. ${ }^{*}$ In comparison with the control group, $p<.05 ;{ }^{*}$ in comparison with the early infection group, $p<.05$; ${ }^{*}$ in comparison with the mid-term infection group, $p<.05$. 


\section{Discussion}

Intrauterine infection refers to the fetal intrauterine infection caused by the invasion of pathogenic microorganisms during the process of pregnancy, and it is one of the important factors for the adverse pregnancy outcomes such as absorbed fetus, abortion, anomaly, premature delivery, dead fetus, intrauterine growth retardation and so on. ${ }^{[11]}$ The pathways of IAI can be classified into ascending infection (through the genital tract), acute maternal infection (hematogenous dissemination through the placenta), retrograde implantation (through the uterine tube from the enterocoelia), amniocentesis and chorionic villus sampling, intrauterine operation and so on. Ascending infection is the most commonly seen route. The sources of infection can be the infection of pathogenic microorganisms in the lower genitourinary tract (e.g., vagina, the neck of uterus), such as trichomonas vaginitis, bacterial vaginosis, colpitis mycotica and so on. It may be caused by direct intrauterine infection, such as chlamydia trachomatis, cytomegalovirus, rubella virus and so on. ${ }^{[12,13]}$ Since it is difficult to make a study on the direct intrauterine infection, this research is designed to adopt the intraperitoneal injection of lipopolysaccharide to establish the IAI animal models on the $3^{\text {rd }}, 9^{\text {th }}$ and $15^{\text {th }}$ day of pregnancy, in order to determine the effect of IAI in different periods. Pyrexia and the up-regulated expression level of inflammatory factors are commonly-seen immune responses, which play an important role in assisting in the anti-infective process. The adverse effects of IAI on the fetal development are considered to be mediated by proinflammatory cytokines to a large extent, and cytokines can go through the placenta and the blood-brain barrier to affect the fetal development. Maternal infection during pregnancy can change the expression of inflammatory factors in the placenta, the amniotic fluid, the fetal brain and lung tissues. The injection of lipopolysaccharide into pregnant rats can activate the inherent immune system and induce the immune cells to release a large number of cytokines (IL-6, IL- $1 \beta$ and TNF- $\alpha$ ) through the mediation of TLR signaling pathway. By inducing the placental inflammatory responses through the placental barrier, these inflammatory factors can induce a series of inflammatory responses in brain and lung tissues in pregnant rats, leading to absorbed fetus, anomaly, premature delivery, dead fetus and so on. ${ }^{[14]}$ The research results showed that, on the $18 \mathrm{~d}$ of pregnancy, the levels of inflammatory factors IL- $1 \beta$, IL- 6 and TNF- $\alpha$ in the serum of pregnant rats, the placental tissues and the fetal brain and lung tissues in IAI groups in different periods were all increased. The earlier the intrauterine infection occurs, the severer the adverse effects on fetal rats will be. Besides, in comparison with the control group, the mean number of fetuses, brain coefficient and lung coefficient were obviously

Published by Sciedu Press lower in the early infection group and the mid-term infection group, meanwhile, the total number and the ratio of dead fetus + absorbed fetus were increased as well; in the late infection group, the mean number of fetuses, brain coefficient and lung coefficient were obviously higher than those in the early infection group and the mid-term infection group, and the total number and the ratio of dead fetus + absorbed fetus were significantly lower. The differences were of statistical significance $(p<.05)$. The above-mentioned results indicate that the earlier IAI occurs in the process of pregnancy, the higher the expression level of inflammatory factors in the serum of the pregnant rats, the placental tissues, the fetal brain and lung tissues will be. It also has a greater effect on the survival rate, the growth and development of fetuses. Placenta is a pregnancy-specific maternal-fetal organ for the exchange of substances. The placenta and the endometrium with normal vascular function can maintain the normal pregnancy, the growth and development of fetus and the onset of labor, and the dysfunction will lead to the abnormality of conception and the abnormal fetal development. ${ }^{[15]}$ The research results showed that, on the $18 \mathrm{~d}$ of pregnancy, the blood vessel density of placenta and endometrium in the early infection group and the mid-term infection group was lower than that in the control group, and this indicator in the late infection group and the mid-term infection group was obviously higher than that in the early infection group. The differences were of statistical significance $(p<.05)$. It is indicated that the earlier occurrence of IAI will lead to a severer damage to the placental and the endometrial blood vessels.

MDA is a type of lipid peroxidative product for the damage brought by free radicals. The measurement of MDA level in brain tissues can reflect the degree of lipid peroxidation and cell injury. MPO is a marker for the activation of the function of neutrophils. The level and the activity of MPO represent the function and the activated state of polymorphonuclear neutrophilic leukocytes. Neutrophils play an important role in combating the invasion of microorganisms and promoting the acute inflammatory responses. However, they can also mediate tissue injury. ${ }^{[16]}$ The research results showed that, in comparison with the control group, the levels of MDA and MPO in brain and lung tissues in the early infection group and the mid-term infection group were obviously increased. However, the above-mentioned indicators in the late infection group were significantly reduced in comparison with the early infection group and the mid-term infection group. The differences were statistically significant $(p<.05)$. It is indicated that the earlier occurrence of IAI has a greater effect on the level of inflammatory factors and the degree of oxidation reactions in the fetal lung tissues. It is because 
the intraperitoneal injection of lipopolysaccharide can lead to inflammatory responses and free radicals and promote the expression of cytokines and adhesion factors, so that polymorphonuclear neutrophilic leukocytes move from vascular endothelial cells to the interstitial space gradually. By self-cascade amplification, polymorphonuclear neutrophilic leukocytes are caused to be amplified, aggravating the brain tissue injury. ${ }^{[17]}$ As this research is still in the phase of animal testing, the influencing mechanism of IAI in different periods on the pregnant rats, the fetal rats and the clinical patients remains to be further studied.

\section{Conclusions}

In summary, IAI can stimulate the increased secretion of cytokines, induce a series of inflammatory responses and damage the placenta and the endometrium. By going through the placental barrier, inflammatory factors can further aggravate the damage of brain, lung and other organs in fetal rats, threatening their lives and the future survival quality. Furthermore, the earlier occurrence of IAI can lead to severer inflammatory responses and tissue injury in pregnant and fetal rats.

\section{Conflicts of InTEREST Disclosure}

The authors declare they have no conflicts of interest.

\section{REFERENCES}

[1] Li L, Wang S. Research Progress on the Mechanism of Multiple Organ Damage in Preterm Neonates Caused by Intrauterine Infection. Medical Recapitulate. 2015; 21(7): 1232-1234.

[2] Cai Q, Lei H, Hu F, et al. Related factors for intrauterine infections in pregnant women during pregnancy. Chinese Journal of Nosocomiology. 2015; 25(18): 4273-4275.

[3] Shcherbina NA, Vygivska LA. Features of local immunity in local inflammatory reactions in pregnant, depending on the implementation of intrauterine infection. Wiadomosci Lekarskie. 2018; 71(1 pt 2): 168-172.

[4] Shen Y, Sun Y, Gu WZ, et al. Intrauterine infection affects early growth and neurobehavioral development in neonatal rats. Journal of Zhejiang University (Medical Sciences). 2019; 48(1): 58-64.

[5] Yang J, Zheng X. Prevention and control of neonatal intrauterine infection. Chinese Journal of Applied Clinical Pediatrics. 2020; 35(11): 814-816. https://doi.org/10.7748/ns.35.4.11.s7

[6] Lv F, Wang L, He B, et al. Research advances in development and function of villous placenta. Journal of Reproductive Medicine. 2012; 21(1): 73-77.

[7] Masaoka N, Nakajima Y, Morooka M, et al. The impact of intrauterine infection on fetal brain damage assessed by S100B protein concentrations in umbilical cord arteries. J Matern Fetal Neonatal Med. 2016; 29(15): 2464-2469. https://doi.org/10.3109/147670 58.2015 .1087501

[8] Liu J, Cheng H, Zhou S, et al. Intraamniotic administration on placental IL-6 and MMP-9 expressions in rats with intrauterine infection of E. coli. Acta Academiae Medicinae Militaris Tertiae. 2008; 30(19): 1803-1806.
[9] Jin S, Lin T, Long W, et al. Changes of febrile response and placental pathological inflammation in pregnant rats after intrauterine infection in late gestation. Chinese Journal of Perinatal Medicine. 2015; 18(3): 222-226.

[10] Sang X. Experimental research on EGFR inhibitors to newborn rats brain injury caused by intrauterine infection. Xinxiang: Xinxiang Medical University; 2015.

[11] Liu Z, Zou L. Research progress on the predictive diagnosis of intrauterine infection. Journal of Clinical and Experimental Medicine. 2016; 15(12): 1238-1241.

[12] Gao Y, Ren H, Dang Z. Expression of MMP-9 in lung injury induced by intrauterine infection in neonatal rats. Journal of Clinical and Experimental Medicine. 2019; 18(16): 1699-1702.

[13] Shi L, Li L, Zhang X. Mechanism by which Etanercept inhibits LPSinduced embryo loss. Current Immunology. 2013; 33(6): 441-445.

[14] Su S, Xiu F, MI J. Effects of Modified Huanglian Wendan Decoction on MDA, SOD and $\mathrm{Na}^{+}-\mathrm{K}^{+}$-ATPase in Cerebral Tissue of Chronic Renal Failure Rats. Shandong Journal of Traditional Chinese Medicine. 2018; 37(6): 513-516.

[15] Zhao N, Li Y, Liu F, et al. Pyroptosis mediated by mitochondrial DNA amplifies the inflammatory response of alveolar macrophage. Chinese Critical Care Medicine. 2018; 30(2): 97-100.

[16] Liu X, Zhu J, Wang J, et al. Clinical analysis of hypertrophic cranial pachymeningitis associated with anti- myeloperoxidase and antineutrophil cytoplasmic antibody with optic nerve dysfunction. Chinese Journal of Neurology. 2020; 53(8): 603-610.

[17] Zhao J, Shao K, Wang Y, et al. The effects of human umbilical cord blood derived mesenchymal stem cells on traumatic brain injury in rats. Immunological Journal. 2015; 31(12): 1042-1045, 1050. 